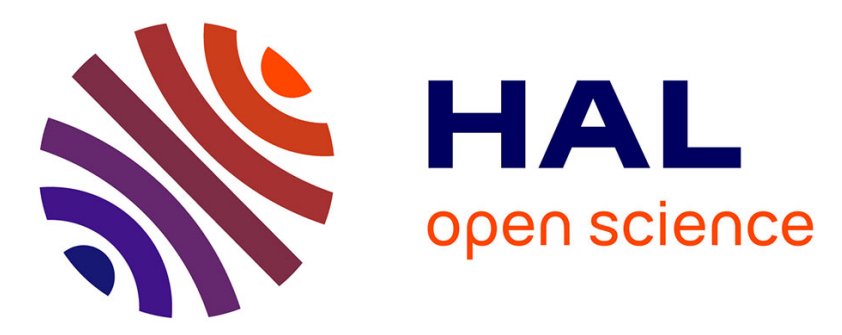

\title{
Structural rigidity optimization of thin laminated shells
}

\author{
A Jibawy, Boris Desmorat, Angela Vincenti
}

\section{To cite this version:}

A Jibawy, Boris Desmorat, Angela Vincenti. Structural rigidity optimization of thin laminated shells.

Composite Structures, 2013, 95, pp.35 - 43. 10.1016/j.compstruct.2012.07.014 . hal-01455391

\section{HAL Id: hal-01455391 \\ https://hal.sorbonne-universite.fr/hal-01455391}

Submitted on 3 Feb 2017

HAL is a multi-disciplinary open access archive for the deposit and dissemination of scientific research documents, whether they are published or not. The documents may come from teaching and research institutions in France or abroad, or from public or private research centers.
L'archive ouverte pluridisciplinaire HAL, est destinée au dépôt et à la diffusion de documents scientifiques de niveau recherche, publiés ou non, émanant des établissements d'enseignement et de recherche français ou étrangers, des laboratoires publics ou privés. 


\title{
Structural rigidity optimization of thin laminated shells
}

\author{
A. Jibawy ${ }^{1,3}$, B. Desmorat ${ }^{1,2}$, A. Vincenti ${ }^{1}$ \\ ${ }^{1}$ IJLRA, UPMC Univ Paris 06 / CNRS UMR 7190, France, \\ \{boris.desmorat,angela.vincenti\}@upmc.fr \\ 2 Univ Paris-Sud 11, France \\ ${ }^{3}$ Segula Technologies, France
}

\begin{abstract}
In this work, we present an optimization methodology used in order to optimize laminated composite shell structures with variable stiffness. Considering the maximization of the structural global rigidity measured by the compliance, a topology optimization problem of the anisotropy fields for thin laminated plates as well as the associated optimization algorithm are extended to thin laminated shells. Numerical examples with quasi-homogeneous angleply stacking sequences showing the optimization methodology feasibility are presented.
\end{abstract}

Keywords: Laminate, Shell, Compliance, Angle ply

\section{Introduction}

Laminated composite shell optimization is a research domain that can be split by considering diverse design objectives and parametrizations related to constant or variable stiffness designs $[1,2]$. Considering the parametrization, two opposite approaches use optimization parameters related to the homogenized shell behaviour (lamination parameters [3, 4], polar parameters [5, 6]) or related directly to the stacking sequence (e.g. by considering the angles of the plies in the stacking sequence).

The weight can be considered as an optimization criterion for shape optimization (shape of the midsurface) or topology optimization (distribution of the thickness) of the shell. A design objective for shell structures may be the

Email address: boris.desmorat@upmc.fr () 
fundamental eigenfrequency $[7,8]$. A third important shell design problem is the maximization of the buckling load $[9,10,11]$. Usually, this problem is related to thin shells, as when the thickness increases, the design objective may become the ply failure or delamination, or more generally damage in the laminated composite [7]. A fifth design objective is the shell structural rigidity optimization $[12,13,14]$. This objective is related to the wish of the smallest variation of the shell shape with respect to one or multiple loadings, e.g. for the use of the shell in an air or liquid flow. This five types of optimization objectives can be considered at the same time if needed by choosing an optimization objective and multiple constraints or by doing multi-criteria optimization [15].

The present paper interest is in variable stiffness design (anisotropy distribution) of laminated composite shell for structural rigidity optimization, assuming that this objective is of greater interest than the others in terms of structure functionality, and that the obtained optimal layup will be verified in order to satisfy buckling and/or damage and/or frequency constraints in a second stage. The proposed optimization methodology is then related to a preliminary design phase (it has to be a very efficient numerical tool) and must be able to treat any 3D surface geometry (and then unstructured meshes).

Considering the compliance as a measure of the global rigidity of the shell structure, a new result demonstrated in this paper is the extension of the compliance optimization problem formulated as a minimum-minimum problem with respect to optimization parameters and statically admissible fields $[16,17]$ to the case of laminated shells, and the definition of the associated minimal set of assumptions. In this framework, the numerical tool used for the examples considers quasi-homogeneous angle-ply stacking sequences: it leads to a very high numerical efficiency while the use of a more general set of stacking sequences is still under study.

In a first section, we introduce the elasticity problem and the variational formulation of a shell structure under the small perturbation assumption, considering symmetric generalized forces and moments and assuming uncoupled behaviour law of the layup. In a second section, we write the optimization problem and algorithm for this shell structure as an extension of a minimum-minimum formulation of compliance optimization. In a third section, we show which assumptions are necessary to use this formulation: the membrane and bending behaviours are uncoupled and the homogenized behaviour does not depend on the shell curvature. In order to derive a semi- 
analytical solution for the sensitivity analysis, we consider specific stacking sequences that are quasi-homogeneous and have isotropic transverse shear behaviour. Finally, two numerical examples are presented: a shell assembly (cylinder with an inner floor) and a 3D elliptic shell geometry with an unstructured mesh.

\section{Thin laminated shell model}

In this section, we introduce necessary notations of the description of the shell geometry. Then we present the elasticity problem in terms of symmetric generalized forces and moments to finally derive the variational formulations used in the optimization problem and algorithm $[18,19]$.

\subsection{Description of the shell geometry}

A point $P$ inside the shell $C$, close to the midsurface $S$, is defined using the point $M$ of the midsurface and the curvilinear coordinates $\left(\xi^{1}, \xi^{2}, \xi^{3}\right)$ :

$$
\mathbf{O P}=\boldsymbol{\Phi}\left(\xi^{1}, \xi^{2}, \xi^{3}\right)=\mathbf{O M}\left(\xi^{1}, \xi^{2}\right)+\xi^{3} \mathbf{a}_{3}\left(\xi^{1}, \xi^{2}\right)
$$

At each point $M$ of the midsurface $S$, the covariant base of the tangent plane to $S$ is defined:

$$
\mathbf{a}_{\alpha}=\frac{\partial \mathbf{O M}\left(\xi^{1}, \xi^{2}\right)}{\partial \xi^{\alpha}} \quad(\alpha=1,2) \quad \mathbf{a}_{3}=\frac{\mathbf{a}_{1} \wedge \mathbf{a}_{2}}{\left\|\mathbf{a}_{1} \wedge \mathbf{a}_{2}\right\|}
$$

This base $\left(\mathbf{a}_{1}, \mathbf{a}_{2}, \mathbf{a}_{3}\right)$ is in general not orthogonal nor normed.

The contravariant base $\left(\mathbf{a}^{1}, \mathbf{a}^{2}, \mathbf{a}^{3}\right)$ of the midsurface is such that

$$
\mathbf{a}_{\alpha} \cdot \mathbf{a}^{\beta}=\delta_{\alpha}^{\beta}, \quad \alpha, \beta=1,2
$$

The second fundamental form (symmetric) of the midsurface $S$, called curvature tensor of $S$, is defined by

$$
\begin{aligned}
\underline{b} & =b_{\alpha \beta} \mathbf{a}^{\alpha} \otimes \mathbf{a}^{\beta} & \text { with } & b_{\alpha \beta}=\mathbf{a}_{\alpha, \beta} \cdot \mathbf{a}_{3} \\
& =b_{\beta}^{\alpha} \mathbf{a}_{\alpha} \otimes \mathbf{a}^{\beta} & \text { with } & b_{\beta}^{\alpha}=\mathbf{a}_{, \beta}^{\alpha} \cdot \mathbf{a}_{3}
\end{aligned}
$$

At each point $P$ of the shell, the $3 \mathrm{D}$ covariant base $\left(\mathbf{g}_{1}, \mathbf{g}_{2}, \mathbf{g}_{3}\right)$ is defined as

$$
\mathbf{g}_{\alpha}=\frac{\partial \mathbf{O P}}{\partial \xi^{\alpha}}=\left(\delta_{\alpha}^{\lambda}-\xi^{3} b_{\alpha}^{\lambda}\right) \mathbf{a}_{\lambda} \quad \mathbf{g}_{3}=\frac{\partial \mathbf{O P}}{\partial \xi^{3}}=\mathbf{a}_{3}
$$


Let's note $\mu$ the determinant of the surface mixed tensor $\mu_{\alpha}^{\lambda}=\left(\delta_{\alpha}^{\lambda}-\xi^{3} b_{\alpha}^{\lambda}\right)$. The elementary volume $d V$ around the point $P$ of the shell is defined by

$$
d V=\mu d \xi^{3} d S
$$

in which $d S=\sqrt{a} d \xi^{1} d \xi^{2}$ is the elementary surface around the point $M$ of the midsurface.

\subsection{Shell local constitutive equations}

The Reissner-Mindlin kinematic assumption reads

$$
\mathbf{U}\left(\xi^{1}, \xi^{2}, \xi^{3}\right)=\mathbf{u}\left(\xi^{1}, \xi^{2}\right)+\xi^{3} \theta_{\alpha}\left(\xi^{1}, \xi^{2}\right) \mathbf{a}^{\alpha} \quad \text { with } \quad \mathbf{u}\left(\xi^{1}, \xi^{2}\right)=u_{i}\left(\xi^{1}, \xi^{2}\right) \mathbf{a}^{i}
$$

The shell model is obtained by a linearisation with respect to $\xi^{3}$ of the strain tensor obtained using the kinematic assumption (8). The linearised strain tensors reads then

$$
\underline{e}=e_{\alpha \beta} \mathbf{g}^{\alpha} \otimes \mathbf{g}^{\beta} \quad \text { with }\left\{\begin{aligned}
e_{\alpha \beta} & =\gamma_{\alpha \beta}(\mathbf{u})+\xi^{3} \rho_{\alpha \beta}(\mathbf{u}, \theta) \quad(\alpha, \beta=1,2) \\
2 e_{\alpha 3} & =\zeta_{\alpha}(\mathbf{u}, \theta) \\
e_{33} & =0
\end{aligned}\right.
$$

in which the components of the membrane strain tensor $\gamma_{\alpha \beta}$, the bending strain tensor $\rho_{\alpha \beta}$ and the shear strain tensor $\zeta_{\alpha}$ are defined by

$$
\begin{aligned}
& \gamma_{\alpha \beta}(\mathbf{u})=\frac{1}{2}\left[\widehat{\Phi}_{\alpha \beta}+\widehat{\Phi}_{\beta \alpha}\right] \quad \text { with } \quad \widehat{\Phi}_{\alpha \beta}=u_{\alpha \mid \beta}-b_{\alpha \beta} u_{3} \\
& \rho_{\alpha \beta}(\mathbf{u}, \theta)=\frac{1}{2}\left[\widehat{\theta}_{\alpha \beta}+\widehat{\theta}_{\beta \alpha}\right] \quad \text { with } \quad \widehat{\theta}_{\alpha \beta}=\theta_{\alpha \mid \beta}-b_{\alpha}^{\nu} \widehat{\Phi}_{\nu \alpha} \\
& \zeta_{\alpha}(\mathbf{u}, \theta)=\theta_{\alpha}+u_{3, \alpha}+b_{\alpha}^{\lambda} u_{\lambda}
\end{aligned}
$$

in which $u_{\alpha \mid \beta}=u_{\alpha, \beta} \Gamma_{\alpha \beta}^{\gamma}\left(\Gamma_{\alpha \beta}^{\gamma}=\mathbf{a}_{\alpha, \beta} \cdot \mathbf{a}^{\gamma}\right.$ are the surface Christoffel symbols).

Let's note $\sigma^{\alpha \beta}$ the components of the stress tensor in the 3D covariant base (at the point $P$ ). The application of the virtual work principle leads to the definition of the symmetric internal generalized stress tensors in membrane $\underline{N}$, in bending $\underline{M}$ and in transverse shear $\mathbf{Q}$. Their components in the covariant base of the midsurface $S$ are

$$
N^{\alpha \beta}=\int_{H} \sigma^{\alpha \beta} \mu \mathrm{d} \xi^{3} \quad M^{\alpha \beta}=\int_{H} \xi^{3} \sigma^{\alpha \beta} \mu \mathrm{d} \xi^{3} \quad Q^{\alpha}=\int_{H} \sigma^{\alpha 3} \mu \mathrm{d} \xi^{3}
$$


in which $H$ denotes the thickness of the shell.

The application of the virtual work principle also leads to the definition of the local equilibrium equations

$$
\begin{array}{ll}
N_{\mid \beta}^{\alpha \beta}-\left[b_{\lambda}^{\alpha} M^{\lambda \beta}\right]_{\mid \beta}-b_{\beta}^{\alpha} Q^{\beta}+p^{\alpha} & =0 \\
M_{\mid \beta}^{\alpha \beta}-Q^{\alpha}+\mathfrak{M}^{\alpha} & =0 \\
Q_{\mid \alpha}^{\alpha}+b_{\alpha \beta} N^{\alpha \beta}-c_{\alpha \beta} M^{\alpha \beta}+p^{3} & =0
\end{array}
$$

and of the boundary conditions in terms of forces on the part $\partial S_{1}$ of the external shape of $S$ :

$$
\left[N^{\alpha \beta}-b_{\lambda}^{\alpha} M^{\lambda \beta}\right] \nu_{\beta}=T^{\alpha} \quad Q^{\alpha} \nu_{\alpha}=T^{3} \quad M^{\alpha \beta} \nu_{\beta}=C^{\alpha}
$$

in which $p^{\alpha} \vec{a}_{\alpha}+p^{3} \vec{a}_{3}$ and $\mathfrak{M}^{\alpha} \vec{a}_{\alpha}$ are the surface forces and moments applied on the midsurface, $\vec{\nu}=\nu_{\alpha} \vec{a}^{\alpha}$ is the normal vector to the external shape of the midsurface, $T^{\alpha} \vec{a}_{\alpha}+T^{3} \vec{a}_{3}$ and $C^{\alpha} \vec{a}_{\alpha}$ are the line forces and moments applied on the part $\partial S_{1}$ of the external shape of the midsurface.

Clamped boundary conditions in terms of displacements on the part $\partial S_{0}$ of the external shape of $S$ are also considered:

$$
u_{i}=0 \quad \theta_{\alpha}=0
$$

\subsection{Laminated shell behaviour law}

Each ply of the laminated shell is made of a linear elastic material with a monoclinic symmetry. The associated behaviour law is

$$
\underline{\sigma}=\underline{\underline{H}}: \underline{e}
$$

The components $H^{i j k l}$ of $\underline{\underline{H}}$ in the $3 \mathrm{D}$ covariant basis (at the point $\mathrm{P}$ ) satisfy the symmetry conditions

$$
H^{i j k l}=H^{k l i j}=H^{j i k l}=H^{i j l k}
$$

and the monoclinic symmetry imply the supplementary conditions

$$
H^{3 \beta \gamma \delta}=H^{333 \delta}=0
$$

Assuming that the transverse stress $\sigma^{33}$ is equal to zero, the behaviour law (17) reads

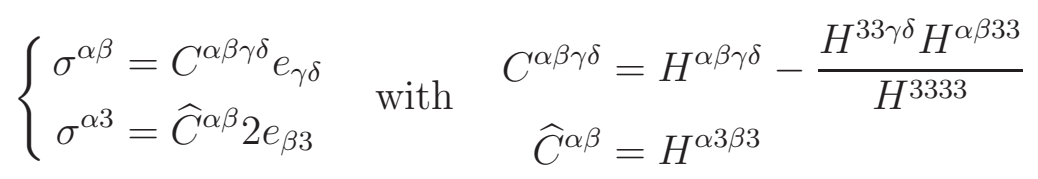


Integrating over the thickness the Eq. (13), the shell behaviour law reads

$$
\underline{N}=\underline{\underline{A}}: \underline{\gamma}+\underline{\underline{B}}: \underline{\rho} \quad \underline{M}=\underline{\underline{B}}: \underline{\gamma}+\underline{\underline{D}}: \underline{\rho}
$$

in which the membrane stiffness $\underline{\underline{A}}$, the coupling stiffness $\underline{\underline{B}}$ and the bending stiffness $\underline{\underline{D}}$ reads

$$
\left\{\begin{aligned}
A^{\alpha \beta \gamma \delta} & =\int_{H} C^{\alpha \beta \gamma \delta} \mu \mathrm{d} \xi^{3} \\
B^{\alpha \beta \gamma \delta} & =\int_{H} C^{\alpha \beta \gamma \delta} \xi^{3} \mu \mathrm{d} \xi^{3} \\
D^{\alpha \beta \gamma \delta} & =\int_{H} C^{\alpha \beta \gamma \delta}\left(\xi^{3}\right)^{2} \mu \mathrm{d} \xi^{3}
\end{aligned}\right.
$$

The transverse shear behaviour law is considered in the following form

$$
\mathrm{Q}=k \underline{F} \cdot \zeta
$$

with

$$
F^{\alpha \beta}=\int_{H} \widehat{C}^{\alpha \beta} \mu \mathrm{d} \xi^{3}
$$

$k$ is the shear coefficient factor, that is assumed to be independent of the shell curvature and of the stacking sequence (this coefficient is equal to $5 / 6$ if we consider a transverse shear behaviour that is homogeneous along the thickness of the shell).

In the behaviour law (21), we will assume through the rest of the paper that the membrane and bending behaviour are uncoupled:

$$
\underline{\underline{B}}=0
$$

(necessary assumptions related to this choice will be presented in section 4.1). The inverse behaviour law reads then

$$
\underline{\gamma}=\underline{\underline{a}}: \underline{N} \quad \underline{\rho}=\underline{\underline{d}}: \underline{M} \quad \boldsymbol{\zeta}=\frac{1}{k} \underline{f} \mathbf{Q}
$$

with

$$
\underline{\underline{a}}=\underline{\underline{A}}^{-1} \quad \underline{\underline{d}}=\underline{\underline{D}}^{-1} \quad \underline{f}=\underline{F}^{-1}
$$

\subsection{Variational formulations}

The elasticity problem $(\mathcal{P})$ is defined by Eq. $(14)(15)(16)(21)(23)$.

Let's define the kinematically admissible displacements field $U_{a d}$, i.e. satisfying the boundary conditions in terms of displacements (16), and the statically admissible stress field $\Sigma_{a d}$, i.e. satisfying the equilibrium equations (14) and the boundary conditions in terms of forces (15). 
Variational formulation in terms of displacements.

If $(\mathbf{u}, \theta)$ is a displacement field solution to the elasticity problem $(\mathcal{P})$ then

$$
\left\{\begin{array}{l}
(\mathbf{u}, \theta) \in U_{a d} \\
\mathfrak{a}\left((\mathbf{u}, \theta),\left(\mathbf{u}^{\prime}, \theta^{\prime}\right)\right)=L\left(\mathbf{u}^{\prime}, \theta^{\prime}\right) \quad \forall\left(\mathbf{u}^{\prime}, \theta^{\prime}\right) \in U_{a d}
\end{array}\right.
$$

with

$$
\begin{aligned}
\mathfrak{a}\left((\mathbf{u}, \theta),\left(\mathbf{u}^{\prime}, \theta^{\prime}\right)\right)= & \iint_{S}\left(A^{\alpha \beta \gamma \delta} \gamma_{\alpha \beta}(\mathbf{u}) \gamma_{\gamma \delta}\left(\mathbf{u}^{\prime}\right)+\ldots\right. \\
& \left.D^{\alpha \beta \gamma \delta} \rho_{\alpha \beta}(\mathbf{u}, \theta) \rho_{\gamma \delta}\left(\mathbf{u}^{\prime}, \theta^{\prime}\right)+k F^{\alpha \beta} \zeta_{\alpha}(\mathbf{u}, \theta) \zeta_{\beta}\left(\mathbf{u}^{\prime}, \theta^{\prime}\right)\right) d S \\
L\left(\mathbf{u}^{\prime}, \theta^{\prime}\right)= & \iint_{S}\left(p^{i} u_{i}^{\prime}+\mathfrak{M}^{\alpha} \theta_{\alpha}^{\prime}\right) d S+\int_{\partial S_{1}}\left(T^{i} u_{i}^{\prime}+C^{\alpha} \theta_{\alpha}^{\prime}\right) d s
\end{aligned}
$$

Complementary energy theorem.

If $(\underline{N}, \underline{M}, \mathrm{Q})$ is a stress field solution to the elasticity problem $(\mathcal{P})$ then

$$
\left\{\begin{array}{l}
(\underline{N}, \underline{M}, \mathbf{Q}) \in \Sigma_{a d} \\
J(\underline{N}, \underline{M}, \mathbf{Q}) \leq J\left(\underline{N^{\prime}}, \underline{M^{\prime}}, \mathbf{Q}^{\prime}\right) \quad \forall\left(\underline{N^{\prime}}, \underline{M^{\prime}}, \mathbf{Q}^{\prime}\right) \in \Sigma_{a d}
\end{array}\right.
$$

with

$$
J(\underline{N}, \underline{M}, \mathbf{Q})=\frac{1}{2} \iint_{S}\left(a_{\alpha \beta \gamma \delta} N^{\alpha \beta} N^{\gamma \delta}+d_{\alpha \beta \gamma \delta} M^{\alpha \beta} M^{\gamma \delta}+\frac{1}{k} f_{\alpha \beta} Q^{\alpha} Q^{\beta}\right) d S
$$

Relation between compliance $L(\mathbf{u}, \theta)$ and complementary energy $J(\underline{N}, \underline{M}, \mathbf{Q})$.

If $(\mathbf{u}, \theta)$ and $(\underline{N}, \underline{M}, \mathbf{Q})$ are solutions to the elasticity problem $(\mathcal{P})$, then

$$
L(\mathbf{u}, \theta)=2 J(\underline{N}, \underline{M}, \mathbf{Q})
$$

\section{Optimization problem and algorithm}

Using the variational formulations of the shell elasticity problem introduced in the previous section in terms of symmetric generalized forces and moments, we introduce the optimization problem and algorithm for the maximization of the shell structural rigidity. This development follows the classical 3D elasticity topology optimization problem [16] and is possible thanks to the assumption of uncoupled behaviour law (25). 


\subsection{Optimization problem}

Structural rigidity optimization of a thin laminated shell subjected to membrane and bending loadings is formulated by the minimization of the compliance with respect to distributed (i.e. continuously variable over the midsurface $S$ of the shell) optimization parameters $\beta_{i} \in\left[\beta_{i}^{\min }, \beta_{i}^{\max }\right]$ :

$$
\min _{\beta_{i} \in\left[\beta_{i}^{\min }, \beta_{i}^{\max }\right]}\left[\iint_{S}\left(p^{i} u_{i}+\mathfrak{M}^{\alpha} \theta_{\alpha}\right) d S+\int_{\partial S_{1}}\left(T^{i} u_{i}+C^{\alpha} \theta_{\alpha}\right) d s\right]
$$

Because the compliance is equal to twice the complementary energy (Eq. (30)), the use of the complementary energy theorem (29) leads to an optimization problem formulated as a double minimization with respect to the optimization parameters and to statically admissible generalized membrane, bending and transverse shear stresses:

$$
\begin{aligned}
& \min _{\beta_{i} \in\left[\beta_{i}^{\text {min }}, \beta_{i}^{\text {max }}\right]} \min _{\left(\underline{N}^{\prime}, \underline{M}^{\prime}, \mathbf{Q}^{\prime}\right) \in \Sigma_{a d}} \\
& {\left[\iint_{S}\left(a_{\alpha \beta \gamma \delta} N^{\prime \alpha \beta} N^{\prime \gamma \delta}+d_{\alpha \beta \gamma \delta} M^{\prime \alpha \beta} M^{\prime \gamma \delta}+\frac{1}{k} f_{\alpha \beta} Q^{\prime \alpha} Q^{\prime \beta}\right) d S\right]}
\end{aligned}
$$

\subsection{Optimization algorithm}

The optimization problem (32) is numerically solved by using the following optimization algorithm [16]:

\section{- Initialization:}

The mesh, the boundary conditions in terms of displacements and forces applied to the shell, and the optimization parameters initial field $\beta_{i}^{(0)}$ are defined in order to calculate using a finite element method the generalized stresses $\left(\underline{N}^{(0)}, \underline{M}^{(0)}, \mathrm{Q}^{(0)}\right)$

\section{- Iteration:}

Each iteration is composed of two parts:

\section{Local minimization with fixed stresses.}

The local criterion is minimized considering fixed stress fields $\left(\underline{N}^{(n)}, \underline{M}^{(n)}, \mathrm{Q}^{(n)}\right)$ by considering the following optimization problem:

$$
\begin{aligned}
\min _{\beta_{i} \in\left[\beta_{i}^{\text {min }}, \beta_{i}^{\text {max }}\right]}\left[\underline{N}^{(n)}: \underline{a}\left(\beta_{i}\right): \underline{N}^{(n)}+\ldots\right. \\
\left.\underline{M}^{(n)}: \underline{\underline{d}}\left(\beta_{i}\right): \underline{M}^{(n)}+\frac{1}{k} \mathbf{Q}^{(n)} \cdot \underline{f}\left(\beta_{i}\right) \cdot \mathbf{Q}^{(n)}\right]
\end{aligned}
$$




\section{Global minimization with fixed optimization parameters.} The elasticity problem $(\mathcal{P})$ associated to the parameters $\beta_{i}^{(n+1)}$ that were determined at the local minimizations step is solved in order to define the new generalized stress fields $\underline{N}^{(n+1)}, \underline{M}^{(n+1)}$ and $\mathbf{Q}^{(n+1)}$.

It is proved that this algorithm is convergent [17]. Its numerical performance is linked to the local sensitivity analyses (no global sensitivity analysis has to be performed).

\section{Laminated thin shell optimization}

In the structural rigidity optimization framework introduced in the previous section, the necessary assumptions in terms of behaviour law and stacking sequences in order to perform shell structural rigidity optimization are defined. Then, the solution of the local minimization problem that leads to the implementation of a numerically efficient optimization tool is derived.

\subsection{About uncoupled behaviour and optimization}

The components $C^{\alpha \beta \gamma \delta}$ and $\widehat{C}^{\alpha \beta}$ in Eq. (22) and (24) are the components of the tensors $\underline{\underline{C}}$ and $\underline{\underline{C}}$ expressed in the $3 \mathrm{D}$ covariant base (i.e. at the point $P)$. They are function of the coordinate $\xi^{3}$ and of curvature radii. Noting with an index $M$ the tensor components expressed at the point $M$ in the covariant base of the midsurface, those components at the point $P$ are linked to the components $C_{M}^{\alpha \beta \gamma \delta}$ and $\widehat{C}_{M}^{\alpha \beta}$ of the tensor $\underline{\underline{C}}$ and $\underline{\underline{C}}$ (at the point $M$ in the covariant base of the midsurface $S$ ) by the following relations

$$
\begin{aligned}
C^{\alpha \beta \gamma \delta} & =m_{\alpha^{\prime}}^{\alpha} m_{\beta^{\prime}}^{\beta} m_{\gamma^{\prime}}^{\gamma} m_{\delta^{\prime}}^{\delta} C_{M}^{\alpha^{\prime} \beta^{\prime} \gamma^{\prime} \delta^{\prime}} \quad\left(\text { with } \quad \mu_{\gamma}^{\alpha} m_{\beta}^{\gamma}=\delta_{\beta}^{\alpha}\right) \\
\widehat{C}^{\alpha \beta} & =m_{\alpha^{\prime}}^{\alpha} m_{\beta^{\prime}}^{\beta} \widehat{C}_{M}^{\alpha^{\prime} \beta^{\prime}}
\end{aligned}
$$

Thus, considering a shell made of an homogeneous isotropic linear elastic material, the behaviour law $(21)$ is coupled $(\underline{B} \neq 0)$. Considering a laminated shell, it is then vain to search in the general case for some particular stacking sequences leading to an uncoupled shell behaviour law.

In order to obtain a uncoupled laminate behaviour law, it is then necessary to neglect the influences of the curvature on $\underline{\underline{A}}, \underline{\underline{B}}$ and $\underline{\underline{D}}$. This leads to assume that

$$
\left.C^{\alpha \beta \gamma \delta} \simeq C^{\alpha \beta \gamma \delta}\right|_{\xi^{3}=0}=C_{M}^{\alpha \beta \gamma \delta} \text { and } \quad \mu=\operatorname{det}\left(\mu_{\alpha}^{\beta}\right) \simeq 1
$$


The components of $\underline{\underline{A}}, \underline{\underline{B}}$ and $\underline{\underline{D}}$ in the covariant base of the midsurface $S$ reads then

$$
\left\{\begin{aligned}
A^{\alpha \beta \gamma \delta} & =\int_{H} C_{M}^{\alpha \beta \gamma \delta} \mathrm{d} \xi^{3} \\
B^{\alpha \beta \gamma \delta} & =\int_{H} C_{M}^{\alpha \beta \gamma \delta} \xi^{3} \mathrm{~d} \xi^{3} \\
D^{\alpha \beta \gamma \delta} & =\int_{H} C_{M}^{\alpha \beta \gamma \delta}\left(\xi^{3}\right)^{2} \mathrm{~d} \xi^{3}
\end{aligned}\right.
$$

Let's note $\left(\mathbf{i}_{1}, \mathbf{i}_{2}, \mathbf{a}_{3}\right)$ the orthonormal base in which is defined the behaviour law (17) in terms of physical components. If one notes $\psi_{\alpha}^{\beta}$ and $\varphi_{\alpha}^{\beta}$ $\left(\psi_{\alpha}^{\gamma} \varphi_{\gamma}^{\beta}=\delta_{\alpha}^{\beta}\right)$ the matrices relating this base to the covariant base of the midsurface $S$ :

$$
\mathbf{a}_{\alpha}=\varphi_{\alpha}^{\beta} \mathbf{i}_{\beta} \quad \mathbf{i}_{\alpha}=\psi_{\alpha}^{\beta} \mathbf{a}_{\beta}
$$

The components in the orthonormal base $\left(\mathbf{i}_{1}, \mathbf{i}_{2}, \mathbf{a}_{3}\right)$ will be noted with a tilde $(\sim)$. It is proved that (see below):

$$
\left\{\begin{aligned}
\tilde{A}^{\alpha \beta \gamma \delta} & =\int_{H} \tilde{C}_{M}^{\alpha \beta \gamma \delta} d \xi^{3} \\
\tilde{B}^{\alpha \beta \gamma \delta} & =\int_{H} \tilde{C}_{M}^{\alpha \beta \gamma \delta} \xi^{3} d \xi^{3} \\
\tilde{D}^{\alpha \beta \gamma \delta} & =\int_{H} \tilde{C}_{M}^{\alpha \beta \gamma \delta}\left(\xi^{3}\right)^{2} d \xi^{3}
\end{aligned}\right.
$$

and the obtained (approximate) shell behaviour laws are identical to those of plates. The uncoupling of membrane/bending behaviour is then obtained with the same stacking sequences than those of laminated plates.

For consistency, we will also neglect the influence of curvature in the transverse shear behaviour (23), that is

$$
\left.\widehat{C}^{\alpha \beta} \simeq \widehat{C}^{\alpha \beta}\right|_{\xi^{3}=0}=\widehat{C}_{M}^{\alpha \beta}
$$

and then

$$
F^{\alpha \beta}=\int_{H} \widehat{C}_{M}^{\alpha \beta} \mathrm{d} \xi^{3} \quad \tilde{F}^{\alpha \beta}=\int_{H} \tilde{\widehat{C}}_{M}^{\alpha \beta} \mathrm{d} \xi^{3}
$$


Proof of Eq. (38).

$$
\begin{aligned}
A^{\alpha \beta \gamma \delta} & =\int_{H} C_{M}^{\alpha \beta \gamma \delta} d \xi^{3}=\int_{H} \psi_{\alpha^{\prime}}^{\alpha} \psi_{\beta^{\prime}}^{\beta} \psi_{\gamma^{\prime}}^{\gamma} \psi_{\delta^{\prime}}^{\delta} \tilde{C}_{M}^{\alpha^{\prime} \beta^{\prime} \gamma^{\prime} \delta^{\prime}} d \xi^{3} \\
& =\psi_{\alpha^{\prime}}^{\alpha} \psi_{\beta^{\prime}}^{\beta} \psi_{\gamma^{\prime}}^{\gamma} \psi_{\delta^{\prime}}^{\delta} \int_{H} \tilde{C}_{M}^{\alpha^{\prime} \beta^{\prime} \gamma^{\prime} \delta^{\prime}} d \xi^{3}
\end{aligned}
$$

by definition $\quad A^{\alpha \beta \gamma \delta}=\psi_{\alpha^{\prime}}^{\alpha} \psi_{\beta^{\prime}}^{\beta} \psi_{\gamma^{\prime}}^{\gamma} \psi_{\delta^{\prime}}^{\delta} \tilde{A}^{\alpha^{\prime} \beta^{\prime} \gamma^{\prime} \delta^{\prime}}$,

$$
\text { then } \tilde{A}^{\alpha \beta \gamma \delta}=\int_{H} \tilde{C}_{M}^{\alpha \beta \gamma \delta} d \xi^{3}
$$

Remark. If the previous assumptions (35) and (39) are not considered in the behaviour law, it is not possible to get an equivalent equation for the relation (38).

\subsection{Choice of specific orthotropic stacking sequences}

Let's consider stacking sequences made of identical plies (identical material in each ply, identical ply thicknesses). The elementary layer is supposed to have an orthotropic linear elastic behaviour. Let's assume that the stacking sequence implies an uncoupled behaviour $(\underline{B}=0)$ and orthotropy in membrane and in bending.

The local minimization problem (33) is analytically solved considering plates in the case of a membrane local energy or of a bending local energy [20]. It is demonstrated that

- 3 cases of optimal orthotropic behaviour are defined with increasing values of $\frac{\sigma_{I}-\sigma_{I I}}{\left|\sigma_{I}+\sigma_{I I}\right|}$ (in which $\sigma_{I}$ and $\sigma_{I I}$ are the two principal stresses, with $\left.\sigma_{I}>\sigma_{I I}\right)$,

- in case 1 : there exist an infinite number of optimal stacking sequences,

- in cases 1 and 2 : the angle-ply is optimal,

- in cases 1,2 and 3: the cross-ply is optimal (and is the only optimal staking sequence in case 3 ).

Considering laminates with identical ply thicknesses, the cross ply is not feasible because a change in relative ply thicknesses will imply a 90 degrees fibre orientation change inside the plies. Considering smooth variations of stress field inside the plate domain, the angle-ply is feasible in the first two cases 
presented above, but is sub-optimal in case 3 and provide discontinuous orientation variations inside plies in the third stress case (the stacking sequence jumps from unidirectional to equilibrated cross-ply at a given value of $\frac{\sigma_{I}-\sigma_{I I}}{\left|\sigma_{I}+\sigma_{I I}\right|}$ that is a function of the elementary layer material parameters).

For shells (or uncoupled plates with simultaneous membrane and bending loadings), the membrane and bending energies coexist and no analytical solution to the local minimization of the sum of membrane and bending energies has been found yet. We will then consider in this work specific stacking sequences in order to solve the local minimization problems.

Introducing the homogenized membrane and bending tensors $\underline{\underline{A}}^{*}=\frac{1}{H} \underline{\underline{A}}$ and $\underline{\underline{D}}^{*}=\frac{12}{H^{3}} \underline{\underline{D}}$, we choose to work with angle-ply stacking sequences that satisfy the quasi-homogeneous property:

$$
\underline{\underline{A}}^{*}=\underline{\underline{D}}^{*}
$$

This quasi-homogeneity property adds an optimization constraint but this difficulty can be easily circumvented if we limit the stacking sequences to those which assure this property [21]. As an example, the following 8 plies stacking sequence is uncoupled, quasi-homogeneous and orthotropic:

$$
[+\alpha /-\alpha /-\alpha /+\alpha /-\alpha /+\alpha /+\alpha /-\alpha]
$$

For this type of stacking sequences, the optimization parameters are the lamination angle $\alpha \in\left[0^{\circ}, 45^{\circ}\right]$ and the stiffness principal direction of orthotropy of the homogenized laminate $\Phi_{1} \in\left[0^{\circ}, 90^{\circ}\right]$.

The transverse shear energy being very small in comparison to those of membrane and bending, we assume that the transverse shear behaviour of the elementary layers is isotropic and has no effect on the local minimization solution. In terms of engineering moduli, this leads to equal shear moduli $G_{T T}$ and $G_{L T}$, and the transverse shear behaviour law (23) reads

$$
\mathrm{Q}=\frac{5}{6} H \underline{\widehat{C}} \cdot \zeta \quad(\underline{F}=H \underline{\widehat{C}})
$$

\subsection{Solution to the local minimization problem}

Considering the assumptions of isotropic transverse shear behaviour (43) and of uncoupled behaviour (25), the local minimization reduces to the minimization of the sum of the membrane and bending energies because the transverse shear energy remains constant during this local minimization with fixed generalized stresses. 
Considering the assumptions (35) and (39) that the homogenized behaviour does not depend on the shell curvature, the part of the optimization criterion that depends on the optimization parameters is written in the orthonormal base $\left(\mathbf{i}_{1}, \mathbf{i}_{2}, \mathbf{a}_{3}\right)$ defined in Eq.(37) and the local minimization problem reads

$$
\min _{\left(\Phi_{1}, \alpha\right)}\left[\frac{1}{H} \tilde{a}_{\alpha \beta \gamma \delta}^{*} \tilde{N}_{\alpha \beta} \tilde{N}_{\gamma \delta}+\frac{12}{H^{3}} \tilde{a}_{\alpha \beta \gamma \delta}^{*} \tilde{M}_{\alpha \beta} \tilde{M}_{\gamma \delta}\right]
$$

in which the homogenized membrane compliance tensor $\underline{\underline{a}}^{*}$ is defined in terms of material parameters only (i.e. does not depend on the shell curvature). Because of the uncoupled behaviour assumption $(25), \underline{\underline{\tilde{a}}}^{*}=\left(\underline{\underline{A}}^{*}\right)^{-1}$, and the membrane and bending homogenized compliance tensors are identical because of the quasi-homogeneity assumption (41).

Missing an analytical solution, we solve numerically this optimization problem using a fixed point algorithm. The choice of multiple initializations allows to find the global optimum in the case of multiple local minima. The numerical cost of the resolution is very low in comparison to a finite element calculation thanks to the existence of semi-analytical solutions to the local minimization problems presented in the next subsections 4.3.1 and 4.3.2.

\subsubsection{Local minimization with respect the principal orthotropy direction with a fixed lamination angle}

The local criterion is written in the following form (expressions of the coefficients $k_{i}$ and $l_{i}$ function of the lamination angle $\alpha$ are given in annex):

$$
\widehat{W}\left(\Phi_{1}\right)=k_{0}+k_{1} \sin 2 \Phi_{1}+k_{2} \cos 2 \Phi_{1}+k_{3} \sin 4 \Phi_{1}+k_{4} \cos 4 \Phi_{1}
$$

The first derivative reads

$$
\frac{\partial \widehat{W}\left(\Phi_{1}\right)}{\partial \Phi_{1}}=2 k_{1} \cos 2 \Phi_{1}-2 k_{2} \sin 2 \Phi_{1}+4 k_{3} \cos 4 \Phi_{1}-4 k_{4} \sin 4 \Phi_{1}
$$

Considering the first derivative equal to zero and taking the square of the previous equation, we get

$$
l_{0}+l_{1} Y+l_{2} Y^{2}+l_{3} Y^{3}+l_{4} Y^{4}=0 \quad \text { with } Y=\cos 2 \Phi_{1}
$$

Considering that $\widehat{W}\left(\Phi_{1}\right)$ is continuous and periodic, the global minimum is found numerically in three steps: 
1. The $Y$ solutions of $(47)$ with $Y \in[0,1]$ are found.

2. Corresponding angles $\Phi_{1}$ solutions not satisfying $\frac{\partial \widehat{W}\left(\Phi_{1}\right)}{\partial \Phi_{1}}=0$ are eliminated.

3. The criterion values for the remaining angles $\Phi_{1}$ are compared in order to find the global minimum.

4.3.2. Local minimization with respect to the lamination angle with a fixed principal orthotropy direction

The local criterion is put in the form (the expressions of the coefficients $\kappa_{i j}$ and $\lambda_{i}$ function of the stiffness principal orthotropy direction $\Phi_{1}$ are given in annex):

$$
\widehat{W}(\alpha)=\frac{1}{H^{3}} \frac{\sum_{i=0}^{4} \kappa_{1 i}(Y)^{i}}{\lambda_{0}+\lambda_{2} Y^{2}+\lambda_{4} Y^{4}} \quad \text { with } \quad Y=\cos (2 \alpha)
$$

The first derivative reads

$$
\frac{\partial \widehat{W}(\alpha)}{\partial \alpha}=-2 \sin (2 \alpha) \frac{\partial \widehat{W}(Y)}{\partial Y} \quad \text { with } \quad \frac{\partial \widehat{W}(Y)}{\partial Y}=\frac{1}{H^{3}} \frac{\sum_{i=0}^{6} \kappa_{2 i}(Y)^{i}}{\left(\lambda_{0}+\lambda_{2} Y^{2}+\lambda_{4} Y^{4}\right)^{2}}
$$

Considering that $\widehat{W}(\alpha)$ is continuous and periodic, the global minimum is found numerically in three steps:

1. $\alpha=0$ is one solution of $\frac{\partial \widehat{W}(\alpha)}{\partial \alpha}=0$.

2. The $Y$ solutions of $\frac{\partial \widehat{W}(Y)}{\partial Y}=0$ with $Y \in[0,1]$ are found.

3. The criterion values for the remaining angles $\alpha$ are compared in order to find the global minimum.

\section{Numerical examples}

\subsection{Finite elements calculation}

In order to simulate numerically thin shell behaviour, we use a mitc4 shell element [18]. The calculation of the through the thickness homogenized 
behaviour law is performed by taking into account the assumptions (35) and (39): the homogenized behaviour does not depend on the shell curvature and the membrane/bending uncoupling is formulated as for plates. This finite element allows to calculate the generalized stress tensors in membrane $\underline{N}$, in bending $\underline{M}$ and in transverse shear $\mathbf{Q}$ in an orthonormal base per element fixed by the user and used to define the material behaviour.

\subsection{Example of a cylinder with an inner floor}

The structure is made of a cylinder with an inside floor diametrally placed in the plane $x=0$. The structure is clamped for $z=0$. An inner pressure is applied on the lower half of the cylinder $(x<0$, Fig. 1). The floor and the cylinder are quasi-homogeneous uncoupled angle-ply laminates with an elementary layer made of Carbon/Epoxy. The optimization procedure is initialized with an unidirectional oriented circumferentially for the cylinder and oriented along the diameter of the cylinder for the floor (Fig. 2). Convergence is obtained after 11 iterations with a relative error of $0.01 \%$. For each iteration, the local minimization step (with 4 initial starting points) and the finite element resolution step (16000 DOFs) take repectively 18 and 5 seconds.

Fig. 3 shows the optimal orientation of the plies oriented at $\Phi_{1}+\alpha$ and $\Phi_{1}-\alpha$. The unidirectional laminate $(\alpha=0)$ is optimal close to the clamped and free edges of the cylinder and of the floor. It gradually changes to an angle-ply with an increasing lamination angle $\alpha$ that increases from 0 to $\pi / 4$. This increase occurs in certain regions in a discontinuous manner, which leads to a straight change from a unidirectional to an equilibrated cross-ply with $\alpha=\pi / 4$ (as it was already mentioned in section 4.2 , this result is not satisfactory from a feasibility point of view).

Table 1 presents the optimization results of the cylinder with a floor. The decrease in terms of compliance and maximal displacements are respectively $85 \%$ and $75 \%$.

\subsection{Example of an elliptic geometry shell}

The structure is made of a $3 \mathrm{D}$ surface with two straight edges and two edges made with a quarter of an ellipse. The structure is clamped on the two straight edges. Considering an unstructured mesh, a constant vertical surface load is applied on the entire surface (Fig. 4). The shell is made of quasi-homogeneous uncoupled angle-ply laminates with an elementary layer made of Carbon/Epoxy. The optimization procedure is initialized with an 
unidirectional oriented from one clamped edge to the second clamped edge (Fig. 5). Convergence is obtained after 34 iterations with a relative error of $0.01 \%$. For each iteration, the local minimization step (with 4 initial starting points) and the finite element resolution step (9000 DOFs) take repectively 14 and 3 seconds.

Fig. 6 shows the optimal orientation of the plies oriented at $\Phi_{1}+\alpha$ and $\Phi_{1}-\alpha$. The unidirectional laminate $(\alpha=0)$ is optimal in some parts of the shell. It gradually changes to an angle-ply with an increasing lamination angle $\alpha$ that increases from 0 to $\pi / 4$. As already noticed in the previous cylinder with an inner floor example, this increase occurs in certain regions in a discontinuous manner, which leads to a straight change from a unidirectional to an equilibrated cross-ply with $\alpha=\pi / 4$.

Table 2 presents the optimization results of the elliptic geometry shell. The decrease in terms of compliance and maximal displacements are respectively $42 \%$ and $50 \%$.

\section{Concluding remarks}

We present in this paper an optimization methodology for laminated shell structures with any arbitrary geometry (the finite element analysis being performed using an unstructured mesh). The optimization algorithm has a great numerical efficiency thanks to the semi-analytical resolution of local sensitivity analysis that has been obtained considering quasi-homogeneous uncoupled angle ply laminates (known to be quasi-optimal for laminated uncoupled plates with membrane only or bending only loadings). Two main assumptions were needed: independence of the homogenized behaviour with the shell curvature and uncoupled membrane and bending behaviours.

The quasi-homogeneous uncoupled angle ply stacking sequences considered are not quite completely adequate for feasibility reasons because there exist some unidirectional to equilibrated cross-ply transitions that cannot be manufactured with laminates made of identical layers. For this reason, a natural perspective to this work would be to consider in a first step an arbitrary uncoupled quasi-homogeneous (or not) homogenized behaviour using for example the polar representation to define the optimization parameters, and to retrieve in a second step feasible stacking sequences made of identical layers which match the optimal distribution of the parameters obtained previously.

[1] Ghiasi H, Pasini D, Lessard L. Optimum stacking sequence design of com- 
posite materials. Part I: Constant stiffness design. Comp Struct 2009;90:111.

[2] Ghiasi H, Fayazbakhsh K, Pasini D, Lessard L. Optimum stacking sequence design of composite materials. Part II: Variable stiffness design. Comp Struct 2010;93:1-13.

[3] Miki M. Material design of composite laminates with required in-plane elastic properties. In: Hayashi T, Kawata K, Umekawa S. editors. Progress in science and engineering of composites, ICCM-IV, Tokyo; 1982. p. $1725-1731$.

[4] Tsai SW. Theory of composite design. Think composites, Dayton, OH, ISBN:0-9618090-3-5; 1992.

[5] Verchery G. In: Proceedings of Colloques EuroMech, Editions du CNRS, vol. 115, 1982. p. 93-104.

[6] Vannucci P. Plane anisotropy by the polar method. Meccanica 1999;40:437-454.

[7] Muc A. Optimal design of composite multilayered plated and shell structures. Thin-Walled Struct 2007;45(10-11):816-820.

[8] Blom AW, Setoodeh S, Hol JMAM, Grdal Z. Design of variable-stiffness conical shells for maximum fundamental eigenfrequency. Comput Struct 2008;86:870-878.

[9] Kicher TP, Chao TL. Minimum weight design of stiffened fibre composite cylinders. J Aircraft 1971;8:562-568.

[10] Walker M, Reiss T, Adali S, Weaver PM. Application of MATHEMATICA to the optimal design of composite shells for improved buckling strength. Eng Comput 1998;15(2):260-267.

[11] Lund E. Buckling topology optimization of laminated multi-material composite shell structures. Comp Struct 2009;91(2):158-167.

[12] Duvaut G, Terrel G, Léné F, Verijenko V. Optimization of fiber reinforced composites. Comp. Struct. 2000;48:83-89. 
[13] Stegmann J, Lund E. Discrete material optimization of general composite shell structures. Int J Numer Methods Eng 2005;62(14):2009-2027.

[14] Stegmann J, Lund E. Nonlinear topology optimization of layered shell structures. Struct Multidiscip Optim 2005;29:249-360.

[15] Abrate S. Optimal design of laminated plates and shells. Compos Struct 1994;29:269-286.

[16] Allaire G, Kohn RV. Optimal design for minimum weight and compliance in plane stress using extremal microstructures. Eur J Mech A-Solids 1993;12:839-878.

[17] Allaire G, Bonnetier E, Francfort G, Jouve F. Shape optimization by the homogenization method. Num. Math. 1997; 76:27-68.

[18] Chapelle D, Bathe KJ. The finite element analysis of shells - Fundamentals. Springer, 2003.

[19] Muller P, Ossadzow C. Introduction aux coques minces élastiques. Hermès, 1999.

[20] Jibawy A, Julien C, Desmorat B, Vincenti A, Léné F. Hierarchical structural optimization of laminated plates using polar representation. Int J Solids Struct 2011; 48(18):2576-2584.

[21] Vannucci P, Verchery G. A special class of uncoupled and quasihomogeneous laminates. Comp Sci Tech 2001;61:1465-1473. 


\section{Annex}

In the local orthonormal base $\left(\mathbf{i}_{1}, \mathbf{i}_{2}, \mathbf{a}_{3}\right)$ (defined in Eq. (37)), the components of the homogenized stiffness tensor $\underline{A}^{*}$, the homogenized compliance tensor $\underline{a}^{*}$, the membrane forces $\underline{N}$ and bending moments $\underline{M}$ are respectively $\tilde{A}_{\alpha \beta \gamma \delta}^{*}, \tilde{\tilde{a}}_{\alpha \beta \gamma \delta}^{*}, \tilde{N}_{\alpha \beta}$ and $\tilde{M}_{\alpha \beta}$. Let $\Phi_{1}$ be the rotation angle of the orthotropy orthonormal base with respect to the local orthonormal base $\left(\mathbf{i}_{1}, \mathbf{i}_{2}, \mathbf{a}_{3}\right)$. In this orthotropy base, the components of the homogenized stiffness tensor, homogenized compliance tensor, membrane forces and bending moments are respectively $A_{\alpha \beta \gamma \delta}^{* \perp}, a_{\alpha \beta \gamma \delta}^{* \perp}, N_{\alpha \beta}^{\perp}$ and $M_{\alpha \beta}^{\perp}$.

Using the polar representation [6], the components of the homogenized stiffness tensor $\stackrel{A^{*}}{=}$ are defined in the local orthonormal base $\left(\mathbf{i}_{1}, \mathbf{i}_{2}, \mathbf{a}_{3}\right)$ by

$$
\begin{aligned}
& \tilde{A}_{1111}^{*}=T_{0}+2 T_{1}+(-1)^{K} R_{0} \cos 4 \Phi_{1}+4 R_{1} \cos 2 \Phi_{1} \\
& \tilde{A}_{1122}^{*}=-T_{0}+2 T_{1}-(-1)^{K} R_{0} \cos 4 \Phi_{1} \\
& \tilde{A}_{1112}^{*}=(-1)^{K} R_{0} \sin 4 \Phi_{1}+2 R_{1} \sin 2 \Phi_{1} \\
& \tilde{A}_{2222}^{*}=T_{0}+2 T_{1}+(-1)^{K} R_{0} \cos 4 \Phi_{1}-4 R_{1} \cos 2 \Phi_{1} \\
& \tilde{A}_{2212}^{*}=\quad-(-1)^{K} R_{0} \sin 4 \Phi_{1}+2 R_{1} \sin 2 \Phi_{1} \\
& \tilde{A}_{1212}^{*}=T_{0} \quad-(-1)^{K} R_{0} \cos 4 \Phi_{1}
\end{aligned}
$$

In the polar representation of $2 \mathrm{D}$ elastic behaviour, five polar components are defined: $T_{0}, T_{1},(-1)^{K} R_{0}, R_{1}$ and $\Phi_{1}$. Four polar components are invariants: $T_{0}$ and $T_{1}$ (linked to the isotropic part of the behaviour) and $(-1)^{K} R_{0}$ and $R_{1}$ (linked to the anisotropic part of the behaviour). The angle $\Phi_{1}$ is the stiffness principal direction of orthotropy.

Using the polar representation, the components of the homogenized compliance tensor $\underline{a}^{*}$ are defined in the local orthonormal base $\left(\mathbf{i}_{1}, \mathbf{i}_{2}, \mathbf{a}_{3}\right)$ by

$$
\begin{aligned}
& \tilde{a}_{1111}^{*}=t_{0}+2 t_{1}+(-1)^{k} r_{0} \cos 4 \varphi_{1}+4 r_{1} \cos 2 \varphi_{1} \\
& \tilde{a}_{1122}^{*}=-t_{0}+2 t_{1}-(-1)^{k} r_{0} \cos 4 \varphi_{1} \\
& \tilde{a}_{1112}^{*}=(-1)^{k} r_{0} \sin 4 \varphi_{1}+2 r_{1} \sin 2 \varphi_{1} \\
& \tilde{a}_{2222}^{*}=t_{0}+2 t_{1}+(-1)^{k} r_{0} \cos 4 \varphi_{1}-4 r_{1} \cos 2 \varphi_{1} \\
& \tilde{a}_{2212}^{*}=-(-1)^{k} r_{0} \sin 4 \varphi_{1}+2 r_{1} \sin 2 \varphi_{1} \\
& \tilde{a}_{1212}^{*}=\begin{array}{lll}
t_{0} & -(-1)^{k} r_{0} \cos 4 \varphi_{1}
\end{array}
\end{aligned}
$$

The polar components of orthotropic stiffness and compliance tensors are 
linked by the relation

$$
\left\{\begin{aligned}
\varphi_{1} & =\Phi_{1}+\frac{\pi}{2} \\
t_{0} & =4 \frac{T_{0} T_{1}-R_{1}^{2}}{\Delta} \\
t_{1} & =\frac{T_{0}^{2}-R_{0}^{2}}{\Delta} \\
(-1)^{k} r_{0} & =4 \frac{R_{1}^{2}-T_{1}(-1)^{K} R_{0}}{\Delta} \\
r_{1} & =2 R_{1} \frac{T_{0}-(-1)^{K} R_{0}}{\Delta}
\end{aligned}\right.
$$

in which

$$
\Delta=16 T_{1}\left(T_{0}^{2}-R_{0}^{2}\right)-32 R_{1}^{2}\left(T_{0}-(-1)^{K} R_{0}\right)
$$

The elementary layer is supposed to be orthotropic. Using the polar representation defined above, the invariant polar components of the elementary layer will be noted $T_{0}^{E L}, T_{1}^{E L},(-1)^{K^{E L}} R_{0}^{E L}, R_{1}^{E L}$. For classical fibre/matrix elementary layers (carbon/epoxy, glass/epoxy, aramid/epoxy), it is experimentally observed that $K^{E L}=0$. For an angle-ply laminate with identical elementary layers (with $K^{E L}=0$ ), it is proved that

$$
\left\{\begin{aligned}
T_{0} & =T_{0}^{E L} \\
T_{1} & =T_{1}^{E L} \\
(-1)^{K} R_{0} & =R_{0}^{E L} \cos 4 \alpha \\
R_{1} & =R_{1}^{E L} \cos 2 \alpha
\end{aligned}\right.
$$

Considering Eq. (51)(52)(53)(54), the components of homogenized compliance tensor of the angle-ply laminate are

$$
\begin{aligned}
& \tilde{a}_{1111}^{*}=V_{1}+V_{2} \cos 2 \Phi_{1}+V_{3} \cos 4 \Phi_{1} \\
& \tilde{a}_{1122}^{*}=V_{4} \quad-V_{3} \cos 4 \Phi_{1} \\
& \tilde{a}_{1112}^{*}=V_{2} \sin 2 \Phi_{1}+V_{3} \sin 4 \Phi_{1} \\
& \tilde{a}_{2222}^{*}=V_{1}-V_{2} \cos 2 \Phi_{1}+V_{3} \cos 4 \Phi_{1} \\
& \tilde{a}_{2212}^{*}=V_{2} \sin 2 \Phi_{1}-V_{3} \sin 4 \Phi_{1} \\
& \tilde{a}_{1212}^{*}=V_{5} \quad-V_{3} \cos 4 \Phi_{1}
\end{aligned}
$$


with $\left\{\begin{array}{l}V_{1}=t_{0}+2 t_{1} \\ V_{2}=-4 r_{1} \\ V_{3}=(-1)^{k} r_{0} \\ V_{4}=-t_{0}+2 t_{1} \\ V_{5}=4 t_{0}\end{array}\right.$ and $\left\{\begin{aligned} t_{0} & =4\left(T_{0}^{\mathrm{EL}} T_{1}^{\mathrm{EL}}-\left(R_{1}^{\mathrm{EL}} \cos 2 \alpha\right)^{2}\right) / \Delta \\ t_{1} & =\left(\left(T_{0}^{\mathrm{EL}}\right)^{2}-\left(R_{0}^{\mathrm{EL}} \cos 4 \alpha\right)^{2}\right) / \Delta \\ (-1)^{k} r_{0} & =4\left(\left(R_{1}^{\mathrm{EL}} \cos 2 \alpha\right)^{2}-T_{1}^{\mathrm{EL}} R_{0}^{\mathrm{EL}} \cos 4 \alpha\right) / \Delta \\ r_{1} & =2 R_{1}^{\mathrm{EL}} \cos 2 \alpha\left(T_{0}^{\mathrm{EL}}-R_{0}^{\mathrm{EL}} \cos 4 \alpha\right) / \Delta\end{aligned}\right.$

in which

$$
\Delta=16 T_{1}^{\mathrm{EL}}\left(\left(T_{0}^{\mathrm{EL}}\right)^{2}-\left(R_{0}^{\mathrm{EL}} \cos 4 \alpha\right)^{2}\right)-32\left(R_{1}^{\mathrm{EL}} \cos 2 \alpha\right)^{2}\left[T_{0}^{\mathrm{EL}}-R_{0}^{\mathrm{EL}} \cos 4 \alpha\right]
$$

The coefficients in Eq. (45),(46) and (47) are derived as following:

$$
\begin{gathered}
k_{i}=\frac{1}{H} p_{1 i}+\frac{12}{H^{3}} p_{2 i} \quad(i=\{0,1,2,3,4\}) \\
\left\{\begin{array}{l}
p_{10}=V_{1}\left(\tilde{N}_{11}^{2}+\tilde{N}_{22}^{2}\right)+2 V_{4} \tilde{N}_{11} \tilde{N}_{22}+V_{5} \tilde{N}_{12}^{2} \\
p_{11}=2 V_{2} \tilde{N}_{12}\left(\tilde{N}_{11}+\tilde{N}_{22}\right) \\
p_{12}=V_{2}\left(\tilde{N}_{11}^{2}-\tilde{N}_{22}^{2}\right) \\
p_{13}=4 V_{3} \tilde{N}_{12}\left(\tilde{N}_{11}-\tilde{N}_{22}\right) \\
p_{14}=V_{3}\left(\tilde{N}_{11}-\tilde{N}_{22}\right)^{2}-4 V_{3} \tilde{N}_{12}^{2}
\end{array}\right. \\
\left\{\begin{array}{l}
p_{20}=V_{1}\left(\tilde{M}_{11}^{2}+\tilde{M}_{22}^{2}\right)+2 V_{4} \tilde{M}_{11} N_{22}+V_{5} \tilde{M}_{12}^{2} \\
p_{21}=2 V_{2} \tilde{M}_{12}\left(\tilde{M}_{11}+\tilde{M}_{22}\right) \\
p_{22}=V_{2}\left(\tilde{M}_{11}^{2}-\tilde{M}_{22}^{2}\right) \\
p_{23}=4 V_{3} \tilde{M}_{12}\left(\tilde{M}_{11}-\tilde{M}_{22}\right) \\
p_{24}=V_{3}\left(\tilde{M}_{11}-\tilde{M}_{22}\right)^{2}-4 V_{3} \tilde{M}_{12}^{2}
\end{array}\right. \\
\left\{\begin{array}{l}
l_{0}=4\left(k_{2}^{2}+4 k_{3}^{2}\right) \\
l_{1}=\left(-16 k_{1} k_{3}+32 k_{2} k_{4}\right) \\
l_{2}=4\left(k_{1}^{2}-k_{2}^{2}-16 k_{3}^{2}+16 k_{4}^{2}\right) \\
l_{3}=32\left(k_{1} k_{3}-k_{2} k_{4}\right) Y^{3} \\
l_{4}=64\left(k_{3}^{2}-k_{4}^{2}\right)
\end{array}\right.
\end{gathered}
$$

and the coefficients in Eq. (48) and (49) are derived using the orthotropy 
base as following:

$$
\left\{\begin{aligned}
& \lambda_{0}=16\left(-\left(R_{0}^{E L}\right)^{2}+\left(T_{0}^{E L}\right)^{2}\right) T_{1}^{E L} \\
& \lambda_{2}=-32\left(R_{1}^{E L}\right)^{2}\left(R_{0}^{E L}+T_{0}^{E L}\right)+64\left(R_{1}^{E L}\right)^{2} T_{1}^{E L} \\
& \lambda_{4}=64 R_{0}^{E L}\left(\left(R_{1}^{E L}\right)^{2}-R_{0}^{E L} T_{1}^{E L}\right) \\
& \kappa_{1 i}=12 \pi_{1 i}+\pi_{2 i} H^{2} \quad(i=\{0,1,2,3,4\}) \\
& \kappa_{20}=+\lambda_{0} \kappa_{11} \\
& \kappa_{21}=-2 \lambda_{2} \kappa_{10}+2 \lambda_{0} \kappa_{12} \\
& \kappa_{22}=-\lambda_{2} \kappa_{11}+3 \lambda_{0} \kappa_{13} \\
& \kappa_{23}=-4 \lambda_{4} \kappa_{10}+4 \lambda_{0} \kappa_{14} \\
& \kappa_{24}=-3 \lambda_{4} \kappa_{11}+\lambda_{2} \kappa_{13} \\
& \kappa_{25}=-2 \lambda_{4} \kappa_{12}+2 \lambda_{2} \kappa_{14} \\
& \kappa_{26}=-\lambda_{4} \kappa_{13}
\end{aligned}\right.
$$

with

$$
\begin{aligned}
& \left\{\begin{aligned}
\pi_{10}= & -2\left(N_{11}^{\perp}+N_{22}^{\perp}\right)^{2}\left(R_{0}^{E L}-T_{0}^{E L}\right)\left(R_{0}^{E L}+T_{0}^{E L}\right) \\
& \left.\quad+4\left[4 N_{12}^{\perp 2}\left(-R_{0}^{E L}+T_{0}^{E L}\right)+\left(N_{11}^{\perp}-N_{22}^{\perp}\right)^{2}\left(R_{0}^{E L}+T_{0}^{E L}\right)\right) T_{1}^{E L}\right] \\
\pi_{11}= & -8\left(N_{11}^{\perp}-N_{22}^{\perp}\right)\left(N_{11}^{\perp}+N_{22}^{\perp}\right) R_{1}^{E L}\left(R_{0}^{E L}+T_{0}^{E L}\right) \\
\pi_{12}= & 8\left[-4 N_{12}^{\perp 2}\left(R_{1}^{E L}\right)^{2}+R_{0}^{E L}\left(\left(N_{11}^{\perp}+N_{22}^{\perp 2}\right) R_{0}^{E L}-\left(-4 N_{12}^{\perp 2}+\left(N_{11}^{\perp}-N_{22}^{\perp}\right)^{2}\right) T_{1}^{E L}\right)\right] \\
\pi_{13}= & 16\left(N_{11}^{\perp}+N_{22}^{\perp}\right)\left(N_{11}^{\perp}-N_{22}^{\perp}\right) R_{0}^{E L} R_{1}^{E L} \\
\pi_{14}= & -8\left(N_{11}^{\perp}+N_{22}^{\perp}\right)^{2}\left(R_{0}^{E L}\right)^{2}
\end{aligned}\right. \\
& \left\{\begin{aligned}
& \pi_{20}=-2\left(M_{11}^{\perp}+M_{22}^{\perp}\right)^{2}\left(R_{0}^{E L}-T_{0}^{E L}\right)\left(R_{0}^{E L}+T_{0}^{E L}\right) \\
&\left.\quad+4\left[4 M_{12}^{\perp 2}\left(-R_{0}^{E L}+T_{0}^{E L}\right)+\left(M_{11}^{\perp}-M_{22}^{\perp}\right)^{2}\left(R_{0}^{E L}+T_{0}^{E L}\right)\right) T_{1}^{E L}\right] \\
& \pi_{21}=-8\left(M_{11}^{\perp}-M_{22}^{\perp}\right)\left(M_{11}^{\perp}+M_{22}^{\perp}\right) R_{1}^{E L}\left(R_{0}^{E L}+T_{0}^{E L}\right) \\
& \pi_{22}=8\left[-4 M_{12}^{\perp 2}\left(R_{1}^{E L}\right)^{2}+R_{0}^{E L}\left(\left(M_{11}^{\perp}+M_{22}^{\perp}\right)^{2} R_{0}^{E L}-\left(-4 M_{12}^{\perp 2}+\left(M_{11}^{\perp}-N_{22}\right)^{2}\right) T_{1}^{E L}\right)\right] \\
& \pi_{23}=16\left(M_{11}^{\perp}+M_{22}^{\perp}\right)\left(M_{11}^{\perp}-M_{22}^{\perp}\right) R_{0}^{E L} R_{1}^{E L} \\
& \pi_{24}=-8\left(M_{11}^{\perp}+M_{22}^{\perp}\right)^{2}\left(R_{0}^{E L}\right)^{2}
\end{aligned}\right.
\end{aligned}
$$




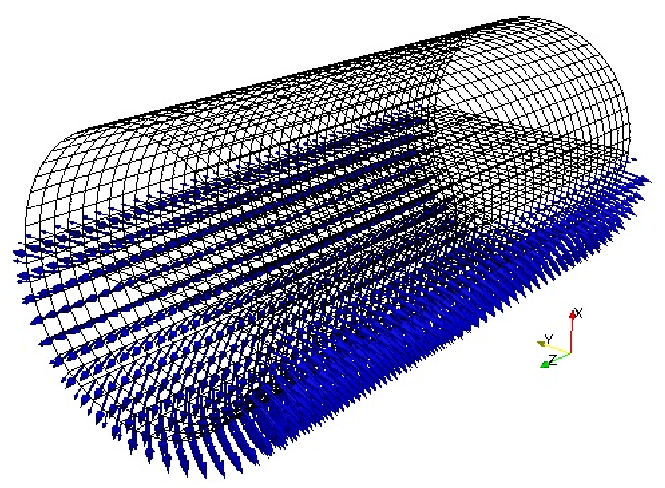

Figure 1: Boundary conditions in terms of forces (cylinder with an inner floor)

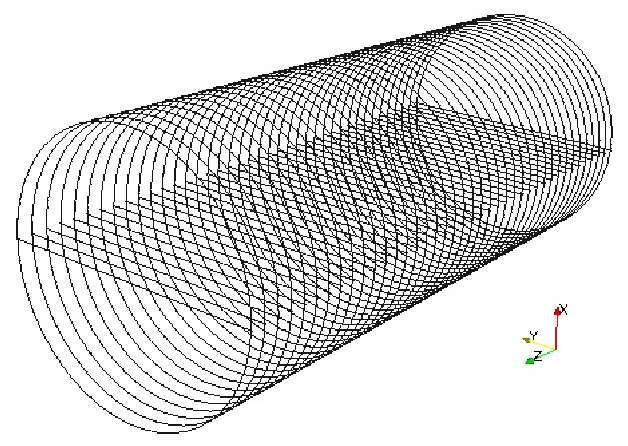

Figure 2: Initial fibre orientation (cylinder with an inner floor)

\begin{tabular}{|c|c|c|c|}
\hline (Normalized values) & Initial state & Optimal state & Decrease \\
\hline Compliance & 100 & 15.0 & $85 \%$ \\
\hline Membrane energy & 95.3 & 10.8 & $89 \%$ \\
\hline Bending energy & 4.6 & 4.1 & $11 \%$ \\
\hline Transverse shear energy & 0.1 & 0.1 & $0 \%$ \\
\hline Max. Displ. & 100 & 25 & $75 \%$ \\
\hline
\end{tabular}

Table 1: Maximal values of normalized criterion and displacements (cylinder with an inner floor) 

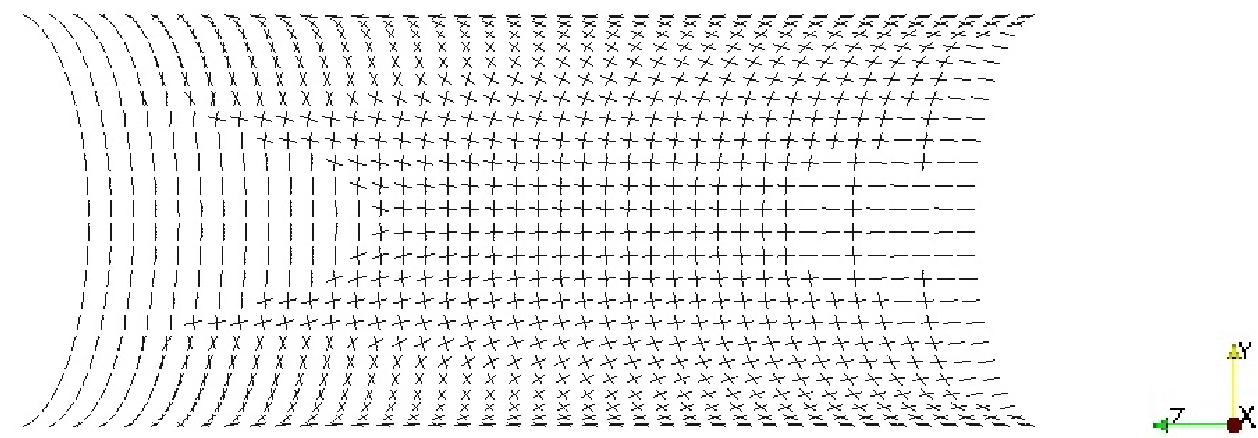

(a) Pressure application zone $(x<0)$

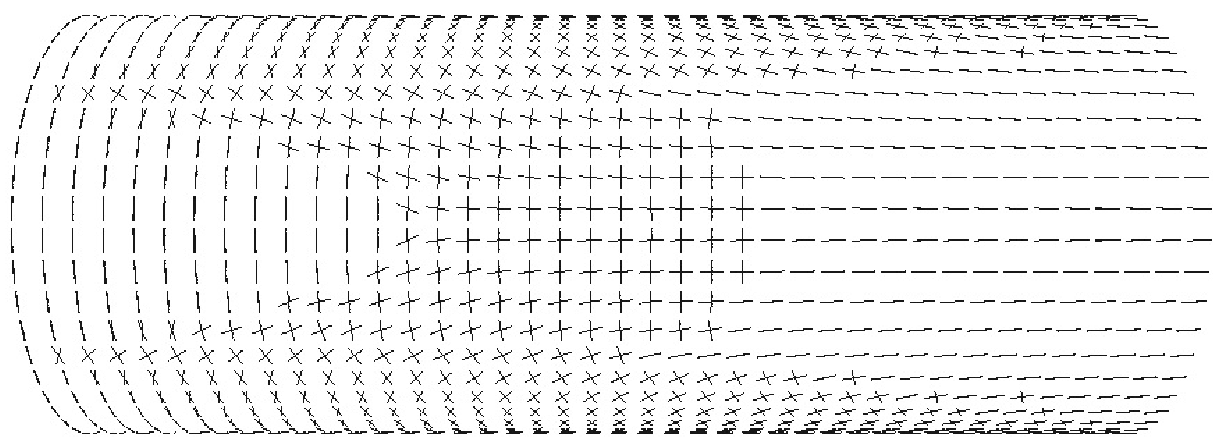

(b) Pressure free cylindrical zone $(x>0)$
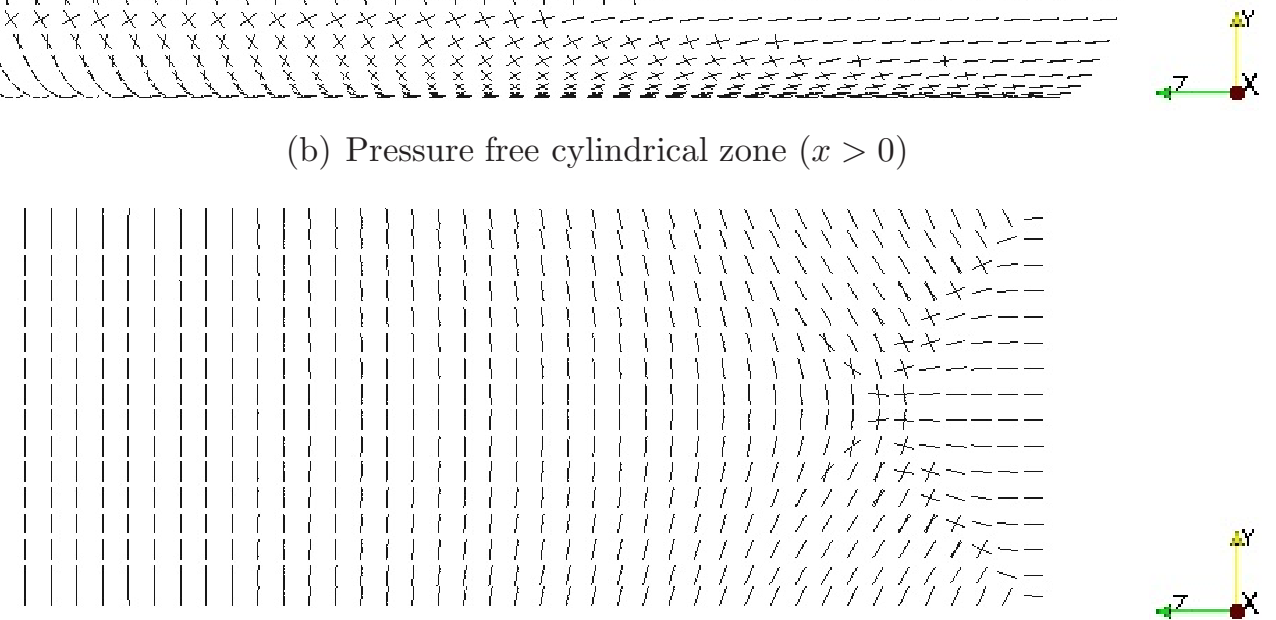

(c) Floor

Figure 3: Optimal plies orientation at the angles $\Phi_{1}+\alpha$ and $\Phi_{1}-\alpha$ (cylinder with an inner floor) 


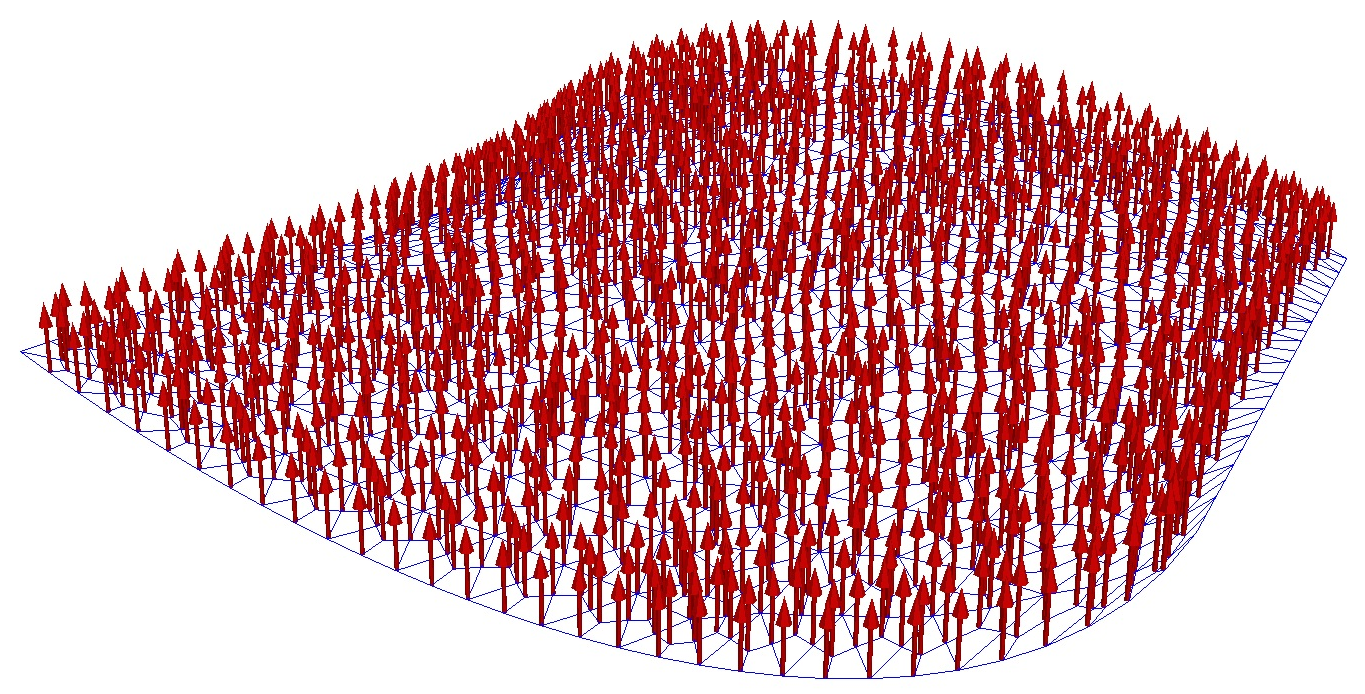

Figure 4: Boundary conditions in terms of forces (elliptic geometry shell)

\begin{tabular}{|c|c|c|c|}
\hline (Normalized values) & Initial state & Optimal state & Decrease \\
\hline Compliance & 100 & 58.2 & $42 \%$ \\
\hline Membrane energy & 62.5 & 35.5 & $43 \%$ \\
\hline Bending energy & 37.2 & 22.4 & $40 \%$ \\
\hline Transverse shear energy & 0.3 & 0.3 & $0 \%$ \\
\hline Max. Displ. & 100 & 50.3 & $50 \%$ \\
\hline
\end{tabular}

Table 2: Maximal values of normalized criterion and displacements (elliptic geometry shell) 


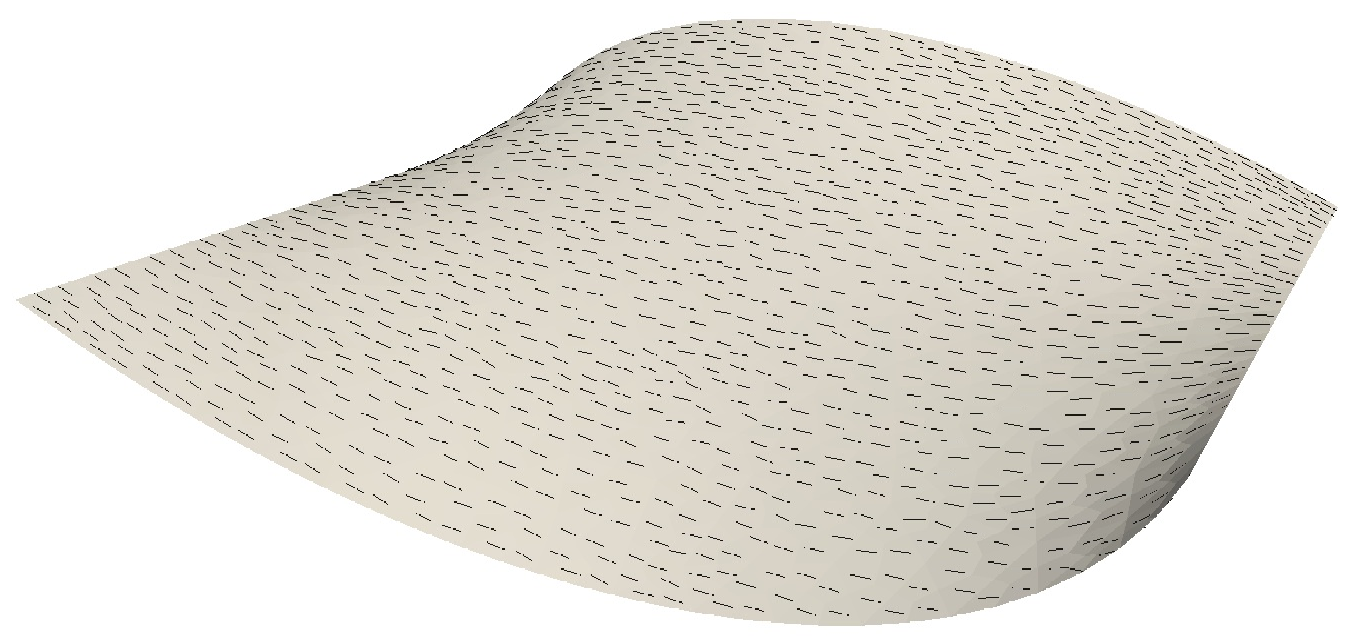

Figure 5: Initial fibre orientation (elliptic geometry shell) 

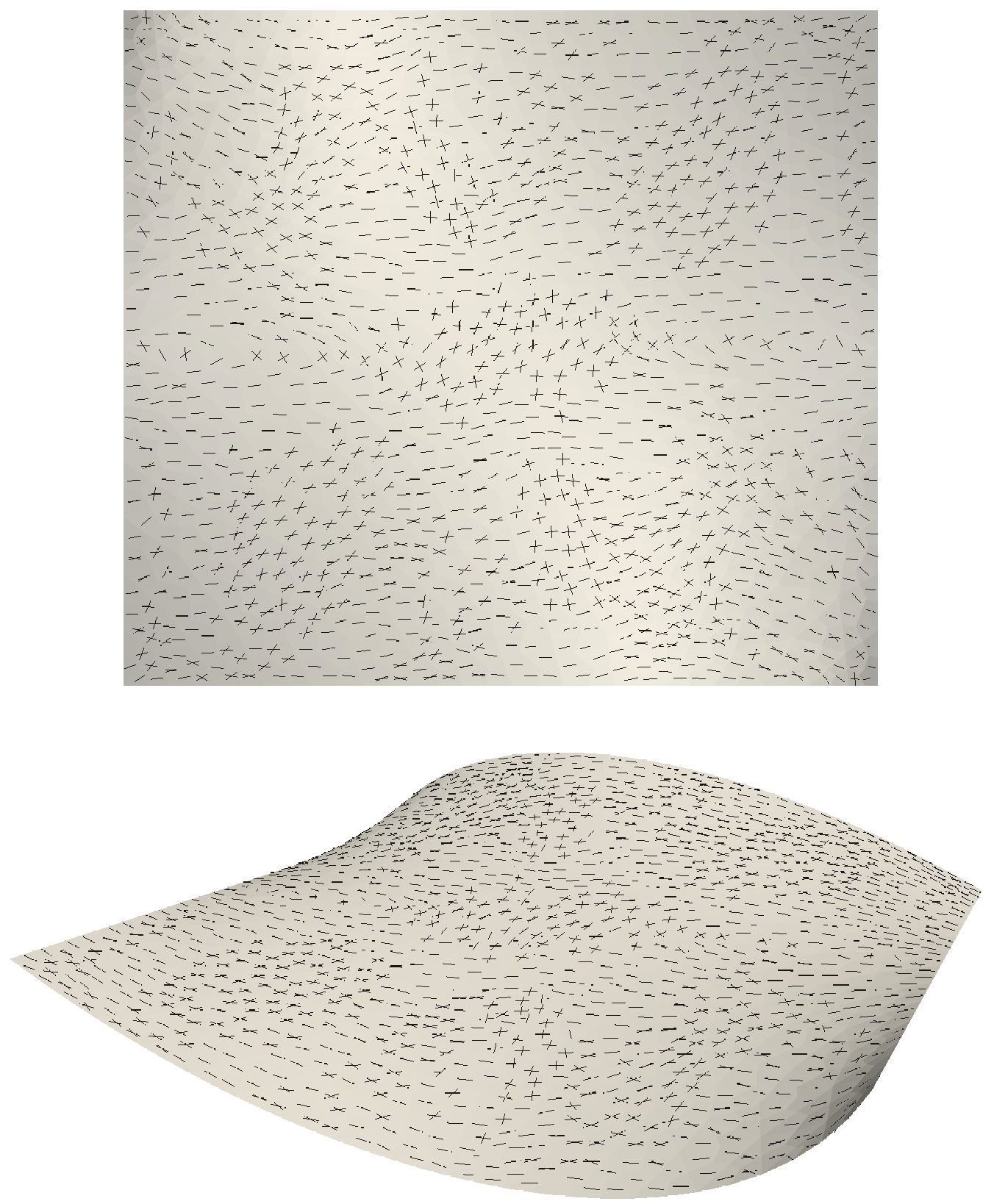

Figure 6: Optimal fibre orientation at the angles $\Phi_{1}+\alpha$ and $\Phi_{1}-\alpha$ (elliptic geometry shell) 